\title{
Nutrient patterns and cardiometabolic risk factors among Iranian adults: Tehran lipid and glucose study
}

\author{
Mohammad Mottaghian ${ }^{1}$, Pantea Salehi ${ }^{2}$, Farshad Teymoori ${ }^{3,4}$, Parvin Mirmiran ${ }^{3 *}$, \\ Firoozeh Hosseini-Esfahani ${ }^{3^{*}}$ (D) and Fereidoun Azizi ${ }^{5}$
}

\begin{abstract}
Background: The present study aimed to assess the relation between nutrient patterns and changes in adult anthropometric and cardiometabolic factors.

Methods: This study was conducted on 1637 adults participating in the Tehran Lipid and Glucose Study (20052008), who were free of cardiovascular diseases and cancer and had completed dietary data. They were followed to the next survey (2008-2011). Dietary intakes were collected and nutrient patterns were obtained. Three year changes in anthropometric and cardiometabolic factors were measured.

Results: Five nutrient patterns were extracted. The first pattern was characterized by "plant protein, thiamine, niacin, and minerals including phosphorus, zinc, copper, magnesium, manganese, and selenium". Animal protein, lactose, vitamin D, riboflavine, pantothenic acid, vitamin B12, calcium, phosphorus, and zinc" were loaded in the second pattern. The third and fourth patterns were characterized by "vitamin K, fiber, calcium, iron, manganese, and potassium", and "high correlation with starch, thiamine and folate, and negative correlation with mono and poly unsaturated fatty acids and vitamin E", respectively. The fifth pattern was high in Fructose, vitamins A, C, pyridoxine, and potassium. There was no association between nutrient patterns and 3-year changes in blood pressure and fasting blood glucose; whereas, per each quartile increment of the fifth pattern adjusted for potential confounders, triglyceride change was decreased $[\beta=-3.66,95 \% \mathrm{Cl}(-6.57,-0.57)$; $P$ for trend $=0.014]$.
\end{abstract}

Conclusion: Present study indicates that nutrient patterns may have an association with cardiometabolic factors, particularly a pattern rich in fructose, vitamins A, C, pyridoxine, and potassium which decreases triglyceride level.

Keywords: Nutrient pattern, Principal component analysis, Fasting blood sugar (FBS), Triglycerides (TGs), High density lipoprotein-cholesterol (HDL), Low density lipoprotein-cholesterol (LDL), Total cholesterol (TC), Blood pressure (BP)

\footnotetext{
* Correspondence: mirmiran@endocrine.ac.ir; f.hosseini@sbmu.ac.ir;

firoozehhosseini@yahoo.com

${ }^{3}$ Nutrition and Endocrine Research Center, Research Institute for Endocrine

Sciences, Shahid Beheshti University of Medical Sciences, P.O. Box:

1985717413, Tehran, Iran

Full list of author information is available at the end of the article
}

(c) The Author(s). 2020 Open Access This article is licensed under a Creative Commons Attribution 4.0 International License, which permits use, sharing, adaptation, distribution and reproduction in any medium or format, as long as you give appropriate credit to the original author(s) and the source, provide a link to the Creative Commons licence, and indicate if changes were made. The images or other third party material in this article are included in the article's Creative Commons licence, unless indicated otherwise in a credit line to the material. If material is not included in the article's Creative Commons licence and your intended use is not permitted by statutory regulation or exceeds the permitted use, you will need to obtain permission directly from the copyright holder. To view a copy of this licence, visit http://creativecommons.org/licenses/by/4.0/. The Creative Commons Public Domain Dedication waiver (http://creativecommons.org/publicdomain/zero/1.0/) applies to the data made available in this article, unless otherwise stated in a credit line to the data. 


\section{Background}

Cardiovascular diseases (CVD) are known as one of the most important causes of mortality and obstacles for sustainable development of human societies in the world [1]. In 2015, the estimated global rate of CVD and its mortality were 422.7 and 17.9 million, respectively [2]. Various risk factors of CVD such as hypertension, dyslipidemia, and hyperglycemia have been recognized previously [3]. Recent studies warn about worldwide trends in increasing blood pressure (BP), body mass index (BMI), serum cholesterol, and blood sugar, particularly in low and middle income countries [4-7].

Dietary factors are one of the main determinants of CVD risk factors. Diet-disease relationships were investigated in the form of dietary patterns from 1980, in this form the pooled effect of whole foods in the diet on different diseases is analyzed [8, 9]. Dietary patterns present a wide view and rational insight of the relation between diet and disease, and can predict the risk of various diseases.

Although dietary patterns based on foods have more applicability in communities, but there are different kinds of foods with various cooking and preservation methods in the world; also, dietary patterns are unable to determine the mechanisms through which main nutrients are related to whole body homeostasis [10]. However, nutrients are dietary components which perform the effects of diet, their composition is the same in the entire world, and not affected by different cooking methods and food preservation [10]. Several recent studies used alternative methods of dietary patterns which pooled effects of various nutrients instead of foods and investigated the association of main dietary nutrient patterns with the risk of chronic disease [11-16]. In this approach dietary intake of nutrients are calculated using food composition databases and then the group of nutrients which have high correlation with each other determines as dietary nutrient patterns using statistical methods [14].

Salehi-Abargouei et al. extracted 3 nutrient patterns related to central obesity in adults; the second pattern (thiamine, niacin, betaine, folate, iron, selenium, and starch) and third pattern (glucose, fructose, sucrose, fiber, $\mathrm{C}$ and $\mathrm{K}$ vitamins, and copper) were associated with lower and higher risk of central obesity, respectively [14]. In another study, two nutrient patters including animal derived nutrients, and also, starch and folate were associated with higher body mass index [12]. Other studies extracted different nutrient patterns related to some chronic diseases and biochemical factors such as hemoglobin A1c (HbA1c) and fasting blood sugar [1517]. However, cohort studies investigating the association of nutrient patterns and prospective changes in cardiometabolic factors are scarce. The current prospective study aimed to investigate the association of nutrient patterns with cardio metabolic risk factors including lipid profiles, BP, and blood sugar in adult participants.

\section{Methods \\ Subjects}

Subjects of this cohort study were selected among Tehran lipid and glucose study (TLGS) participants, an ongoing population-based prospective study with 3-year interval measurements which began in 1991 in Tehran, the capital of Iran [18].

Participants who aged 30-75 years entered in the third survey (2005-2008), and had complete dietary data were entered the study and followed up for 3-years to the fourth survey (2011-2014). Subjects who had chronic disease, special diet or consumed anti-hypertensive, antihyperglycaemic, and lipid lowering drugs, over- and under-reporters of energy intake, pregnant and lactating women, and who had missing data on measurements at baseline or follow up were excluded from the study participants..

\section{Dietary intake assessment}

Dietary intakes were assessed using a valid and reliable semi-quantitative food frequency questionnaire (FFQ) which previously validated for the TLGS study. The reliability and validity of the FFQ has been previously reported [19]. During face-to-face interview by trained and experienced dieticians, the consumption frequency of each food item during the previous year was collected on a daily, weekly, or monthly basis. Portion sizes of consumed foods, reported in household measures were then converted into grams. The dietary intakes of energy and nutrients were computed using the United States Department of Agriculture (USDA) food composition table (FCT), and the Iranian FCT only for local food items that were not available in USDA FCT.

\section{Physical activity assessment}

The modified physical activity questionnaire which previously validated for participants of the TLGS study was used for assessing physical activity levels. The validity and reliability of this was reported earlier [20]; Interviewers asked participants to report the frequency and time spent on activities of light, moderate, hard, and very hard intensity during the past 12 months, according to a list of common activities of daily life. Physical activity levels were expressed as metabolic equivalent hours per week.

\section{Clinical and biological measurements}

Trained interviewers collected information on age, sex, medical history, medication use, and smoking habits using pretested questionnaires. The participant's weight, height, waist circumference (WC), were measured based 
on standard protocols as previously described [21]. Body mass index (BMI) was calculated as weight (Kg) divided by height squared $\left(\mathrm{m}^{2}\right)$.

After a 12-14-h overnight fasting, between 7:00 and 9: 00 a.m., blood samples were taken while subjects were in a sitting position, then transferred into vacutainer tubes. Blood samples were centrifuged within 30 to $45 \mathrm{~min}$ of collection. Details of the biochemical analyses and measurements including triglycerides (TGs), Serum total cholesterol (TC), high-density lipoprotein-cholesterol (HDL-C), and low density lipoprotein cholesterol (LDL$\mathrm{C}$ ), fasting blood sugar (FBS), and blood pressure (BP) have been reported previously $[18,20,21]$.

\section{Statistical analysis}

Data analyses were conducted using the Statistical Package for Social Sciences (version 20.0; SPSS Inc., Chicago $\mathrm{IL})$. The normality of variables was assessed using histogram charts and Kolmogorov-Smirnov analysis. Baseline characteristics of subjects were expressed as mean \pm SD or median (25-75 interquartile range) for continuous variables, and percentages for categorical variables.

The 36 main nutrient intakes of participants at baseline of study were chosen based on their consumption values and literature reviews on their potential association with cardiometabolic factors. Nutrient patterns were derived using principal component analysis (PCA) with varimax rotation and correlation matrix at baseline. Statistical correlation between variables and adequacy of sample size was tested, using the Bartlett test of sphericity $(P<0.001)$ and the Kaiser-Mayer-Olkin test $(0.70)$. Factor scores of each extracted factors were calculated by summing the frequency of consumption multiplied by factor loadings across 36 nutrient items. We identified five dominant factors based on scree plot (Fig. 1) (eigenvalue $>2$ ) and categorized factor scores into quartiles. The 3 year changes of FBS, SBP, DBP, serum TG, HDL-C, LDL-C, and TC were computed by subtracting follow-up from their baseline values. Multiple linear regression analysis was conducted with FBS, BP, and lipid profile changes as dependent variables and quartiles of nutrient patterns as independent variables.

Analysis was conducted using three models of adjustment for potential confounders. The first model was adjusted for age and sex. The second was adjusted for model 1 and BMI, physical activity, and smoking. And the last model includes model 2 and daily energy intake, education levels, marital and employment status. Furthermore, for each of dependent variables, the corresponding value in the baseline survey was adjusted. Data were analysed using SPSS statistics software version 20 . Beta coefficients (unstandardized) and their respective confidence intervals $95 \%(95 \% \mathrm{CI})$ were reported, and $P$ values $<0.05$ were considered as statistically significant.

\section{Results}

Of 12,523 participants who entered in the third survey (2005-2008), 3462 were randomly selected and agreed to complete the dietary assessment, of these, 2101 participants aged 30-75 years were included and followed up to the fourth survey (2011-2014) (Fig. 1). After applying the exclusion criteria, 1803 participants enrolled for the study at baseline; they were followed for 3 years and 166 subjects had missing data on the follow-up assessments. Finally, 1637 participants remained for the final analysis (follow up rate, 90.7\%).

The mean age and BMI of participants (45.8\% male) were 46.6 years and $28.0 \mathrm{Kg} / \mathrm{m} 2$, respectively. The median (IQR, 25, 75) of 3 years changes of SBP and DBP were $3.0(-5.0,11.0)$ and $3.0(-2.0,10.0) \mathrm{mm} \mathrm{Hg}$, respectively. Furthermore, the median (IQR, 25, 75) change values for FBS, TG, HDL, LDL, and TC were 7.0 (1.0, 13.0), - 3.0 (-36.0, 26.0), 5.0 (1.0, 9.0), - 3.2 (-17.8, 11.8), $2.0(-16.0,18.0) \mathrm{mg} / \mathrm{dl}$, respectively.

Table 1 shows factor loading matrix of 36 nutrients and explains variances of each of five nutrient patterns. Using factor analysis, five dominant patterns (Fig. 2) were identified which explained $62 / 2 \%$ of total variations of 36 main nutrient intakes. The first pattern, characterized by plant proteins, thiamine, niacin and minerals including phosphorus, zinc, copper, magnesium, manganese and selenium had $22 \%$ coverage of total variance. The nutrients including animal protein, lactose, vitamin $\mathrm{D}$, riboflavin, pantothenic acid, vitamin B12, calcium, phosphorus and zinc were highly loaded in the second pattern. The third pattern had the highest loading for vitamin $\mathrm{K}$, fiber, calcium, iron, manganese and potassium. The fourth pattern had positive correlation with starch, thiamine and folate, and high negative correlation with mono and poly-unsaturated fatty acids and vitamin $\mathrm{E}$. The fifth pattern was characterized by fructose, vitamins $A$ and $C$, pyridoxine and potassium.

General characteristics of participants based on quartiles of nutrient patterns are shown in Table 2. Across quartiles of the first pattern, the mean age, male percentage, BMI and WC, FBS, SBP, DBP, and TGs increased $(P<0.05)$. Also, for the second pattern, HDL-C increased across quartiles 1 to 4 ; while, male percentage and smokers reduced $(P<0.001)$. With increasing quartiles of the third pattern, the mean of FBS increased and male percentage decreased $(P<0.05)$. Across quartiles of the fourth pattern, the percentage of men, mean age and SBP of participants increased and BMI decreased $(P<$ 0.05). The mean age of participants, BMI, SBP and HDL-C across quartiles of the fifth pattern increased $(P<0.05)$; however, the percentage of smokers, men, married individuals, high education levels, and the mean of TGs decreased $(P<0.05)$.

Table 3 showed dietary intake of macronutrients and food groups across quartiles of nutrient patterns. With 


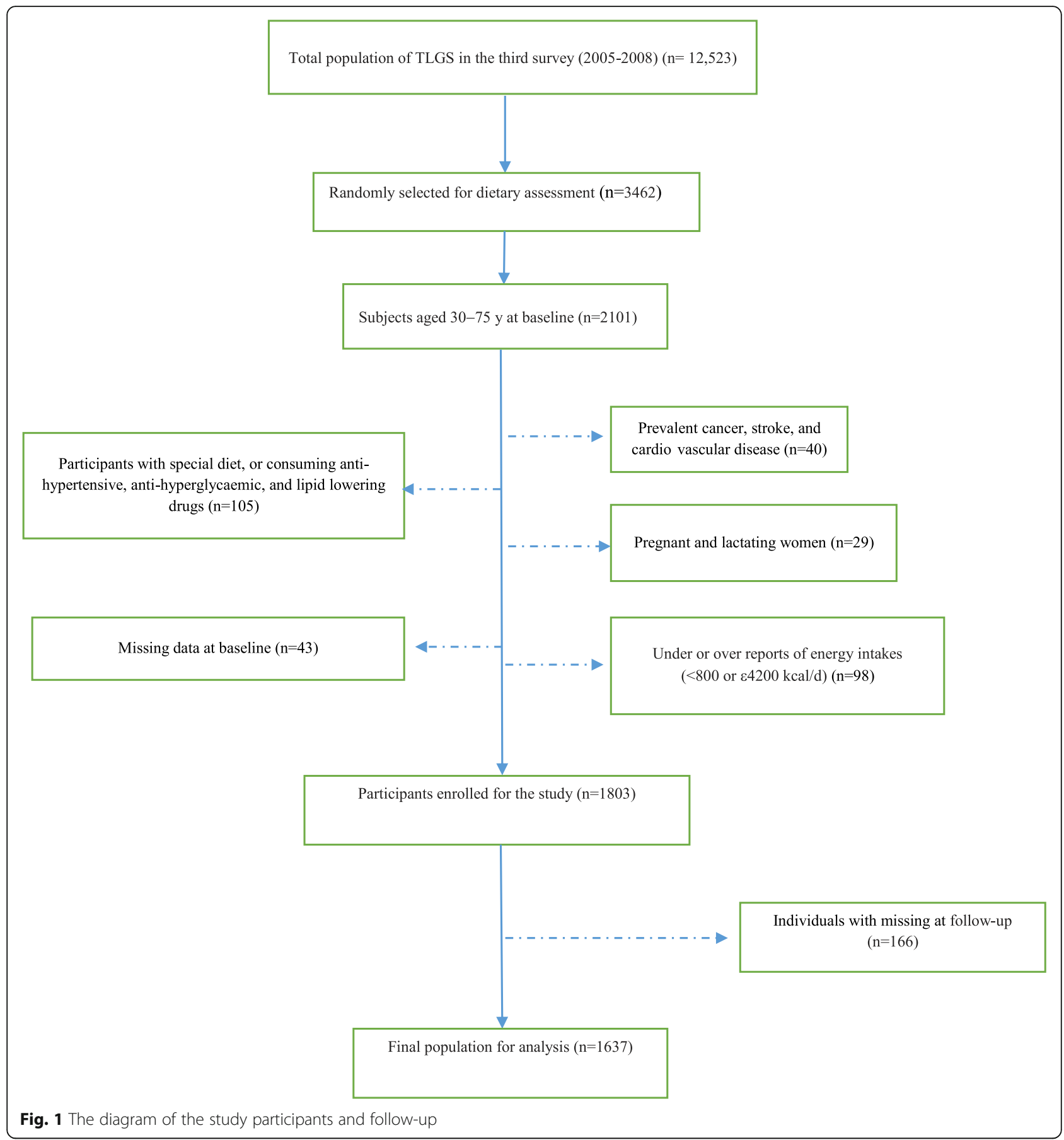

increasing quartiles of the first pattern, carbohydrate, protein, fiber, grain, vegetable, white meat, and legume intakes increased $(P<0.001)$; whereas, fat, fruit, dairy and simple sugar consumptions decreased $(P<0.001)$. Across quartiles of the second pattern energy intake, protein, fat, fruit, vegetable, dairy, red meat and legume consumption increased $(P<0.05)$; however, the intake of carbohydrate, fiber, grain and simple sugar decreased $(P<0.05)$. Dietary protein, fiber, vegetable, dairy, white meat, and legume increased across quartiles of the third pattern $(P<0.05)$; whereas, grain and simple sugar intakes decreased $(P<0.05)$. With increasing quartiles of the fourth pattern, intake of carbohydrate, protein, fiber, grain, fruit, and dairy products increased $(P<0.05)$; however, fat, vegetable, red meat, and legume decreased $(P<0.05)$. Dietary intake of carbohydrate, protein, fiber, fruit, vegetable, and legume increased across quartiles of the fifth pattern 
Table 1 Factor loading matrix and explained variances for major nutrient patterns identified by factor analysis in 1637 participants aged 30-75 years of Tehran Lipid and Glucose Study (2006-2008) a b

\begin{tabular}{|c|c|c|c|c|c|}
\hline \multicolumn{6}{|l|}{ Nutrient patterns } \\
\hline & Pattern 1 & Pattern 2 & Pattern 3 & Pattern 4 & Pattern 5 \\
\hline \multicolumn{6}{|l|}{ Nutrients } \\
\hline Starch & & & & 0.40 & \\
\hline \multicolumn{6}{|l|}{ Sucrose } \\
\hline Lactose & & 0.90 & & & \\
\hline Fructose & & & & & 0.55 \\
\hline \multicolumn{6}{|l|}{ Glucose } \\
\hline Animal protein & & 0.52 & & & \\
\hline Plant protein & 0.80 & & & & \\
\hline \multicolumn{6}{|l|}{ Fiber } \\
\hline Saturated fatty acids & & & 0.40 & & \\
\hline Mono unsaturated fatty acids & & & & -0.84 & \\
\hline Poly unsaturated fatty acids & & & & -0.83 & \\
\hline \multicolumn{6}{|l|}{ cholesterol } \\
\hline Vitamin A & & & & & 0.64 \\
\hline Vitamin D & & 0.73 & & & \\
\hline Vitamin $\mathrm{E}$ & & & & -0.78 & \\
\hline Vitamin $\mathrm{K}$ & & & 0.94 & & \\
\hline Thiamine & 0.55 & & & 0.53 & \\
\hline Riboflavine & & 0.75 & & & \\
\hline Niacin & 0.52 & -0.32 & & & 0.38 \\
\hline Pantothenic acid & & 0.64 & 0.32 & & 0.46 \\
\hline Pyridoxine & & & 0.36 & 0.58 & \\
\hline \multicolumn{6}{|l|}{ Folate } \\
\hline Vitamin B12 & & 0.41 & & & \\
\hline Vitamin C & & & 0.64 & & 0.86 \\
\hline Calcium & & 0.71 & & & \\
\hline Phosphor & 0.42 & 0.72 & & & \\
\hline Iron & & & 0.93 & & \\
\hline Zinc & 0.60 & 0.42 & 0.32 & & \\
\hline Copper & 0.66 & & & & \\
\hline Magnesium & 0.75 & & & & \\
\hline Manganese & 0.66 & & 0.51 & & \\
\hline \multicolumn{6}{|l|}{ Chromium } \\
\hline Selenium & 0.88 & & & & \\
\hline \multicolumn{6}{|l|}{ Sodium } \\
\hline Potassium & & 0.38 & 0.55 & & 0.67 \\
\hline \multicolumn{6}{|l|}{ Caffeine } \\
\hline Explained variance (\%) & 22.0 & 17.2 & 9.5 & 6.8 & 6.4 \\
\hline Cumulative explained variance (\%) & 22.0 & 39.3 & 48.8 & 55.7 & 62.2 \\
\hline
\end{tabular}

aprinciple Component Analysis (PCA) performed on 36 nutrients adjusted for total energy intake

Nutrients with loadings $>0.40$ and less than -0.40 (in bold) are being characteristic for the five patterns; loadings less than 0.3 (in absolute value) are suppressed ${ }^{\mathrm{b}}$ Kaiser's Measure of Sampling Adequacy, $\mathrm{KMO}=0.70$, Bartlett's test of sphericity $=<0.001$ 


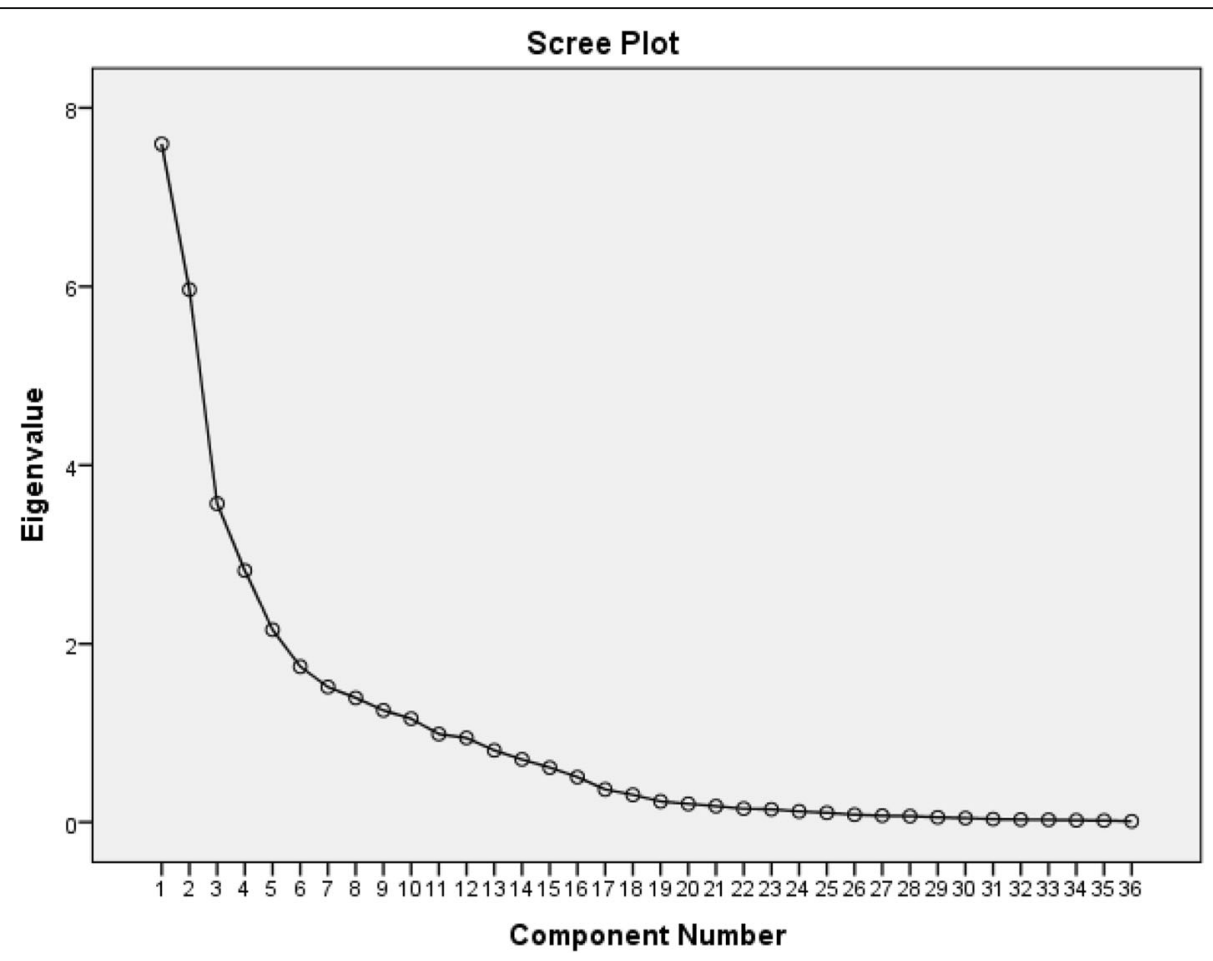

Fig. 2 Scree plot for extraction of dietary nutrient patterns by principal component analysis. The 36 dietary nutrients was used as input variables and nutrient patterns based on eigenvalues $>2$ were identified as main nutrient patterns

$(P<0.05)$; whereas, intake of energy, fat, grain, red meat and simple sugar decreased $(P<0.001)$.

The association of nutrient patterns and 3 year changes in FBS, SBP, and DBP are shown in Table 4. There were no associations between quartiles of nutrient patterns with changes in FBS, SBP, and DBP in crude and adjusted models.

Table 5 shows the association between nutrient patterns and 3-year changes in lipid profiles. In age and sex adjusted model, per each quartile increment of the fifth nutrient pattern, the beta $(\beta)$ coefficient $(95 \% \mathrm{CI})$ of TGs change was $-3.40(-6.30,-0.49)$; $P$ for trend $=0.022$. In the final adjusted model, the beta $(\beta)(95 \% \mathrm{CI})$ of TG change was $-3.66(-6.57,-0.57)$; $\mathrm{P}$ for trend $=0.014$. The present study showed no statistically significant relation between other nutrient patterns and the serum lipid profiles.

\section{Discussion}

In this 3-year prospective study among participants of the TLGS, we identified five nutrient patterns using principal component analysis. We observed no association between nutrient patterns with prospective changes in FBS, SBP, DBP, HDL, LDL, and TC. However, the risk of TG change decreased in participants who had higher adherence to the fifth pattern, which was characterized by fructose, vitamins $\mathrm{A}$ and $\mathrm{C}$, pyridoxine and potassium.

Nutrient patterns and prospective changes in BP and FBS To our knowledge, this study was the first to investigate the association of nutrient patterns with prospective changes in FBS and BP, and observed no significant association. However, the relation between nutrient patterns extracted from factor analysis and serum glucose and BP levels investigated in some cross-sectional studies $[15,16]$. Chikowore et al. conducted a gender base analysis on 2010 urban and rural black participants from South Africa, and identified three nutrient patterns [16]. The pattern characterized by complex carbohydrates, fiber, and B group vitamins, and a pattern which was rich in thiamin, zinc and plant proteins were associated with lower serum glucose and HbA1c, in rural women and men, respectively. Differences in study populations, races, and study design are considerable factors which could justify inconsistent findings of these two studies.

At baseline of our study, participants who had higher adherence to the first, fourth, and fifth nutrient patterns had higher unadjusted values of SBP levels. Also across quartiles of the first and third patterns, the FBS values increased. However, in our study patterns did not make significant changes in FBS and BP after 3-years of follow up after adjusting for potential confounders; It is 


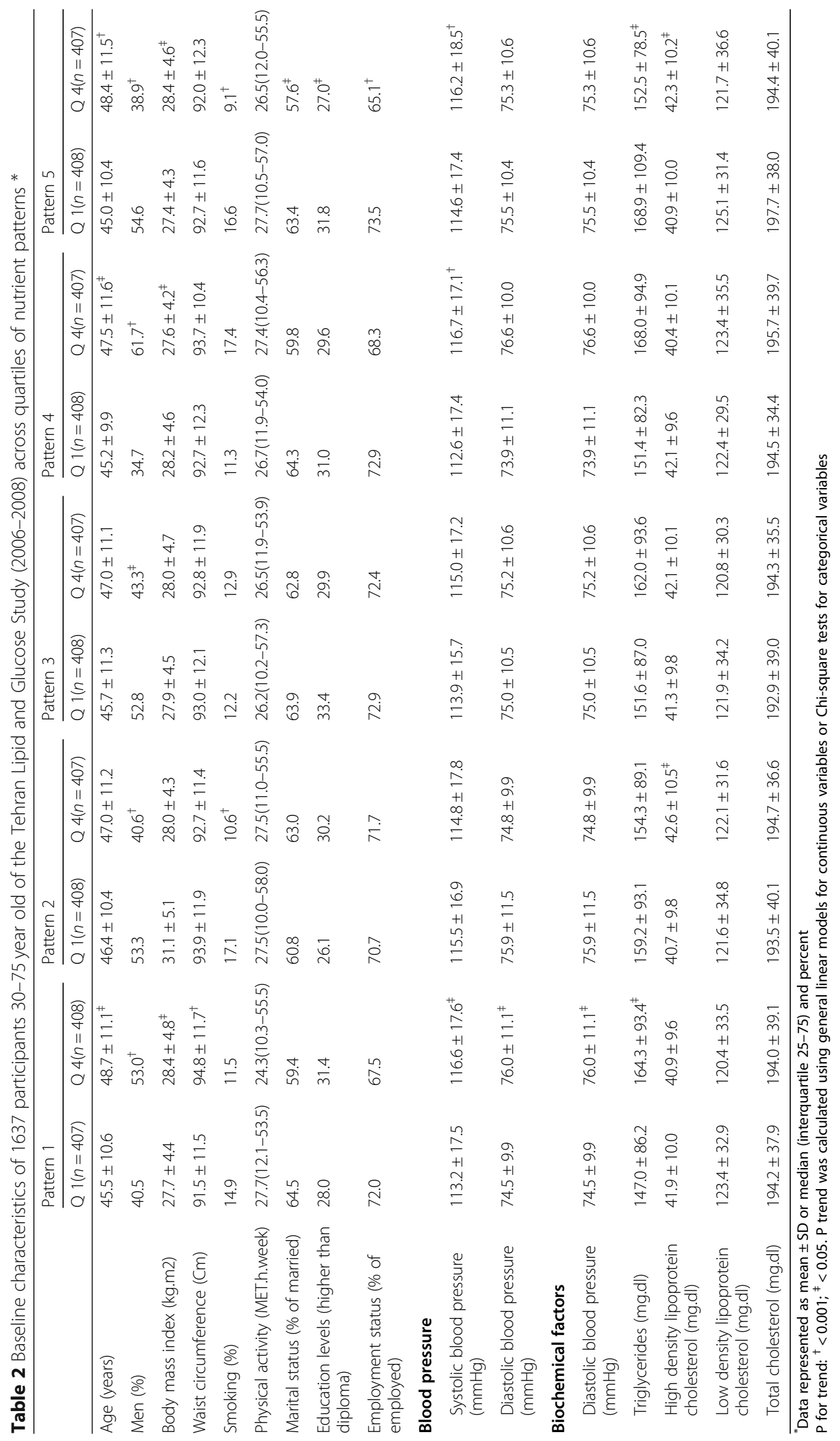




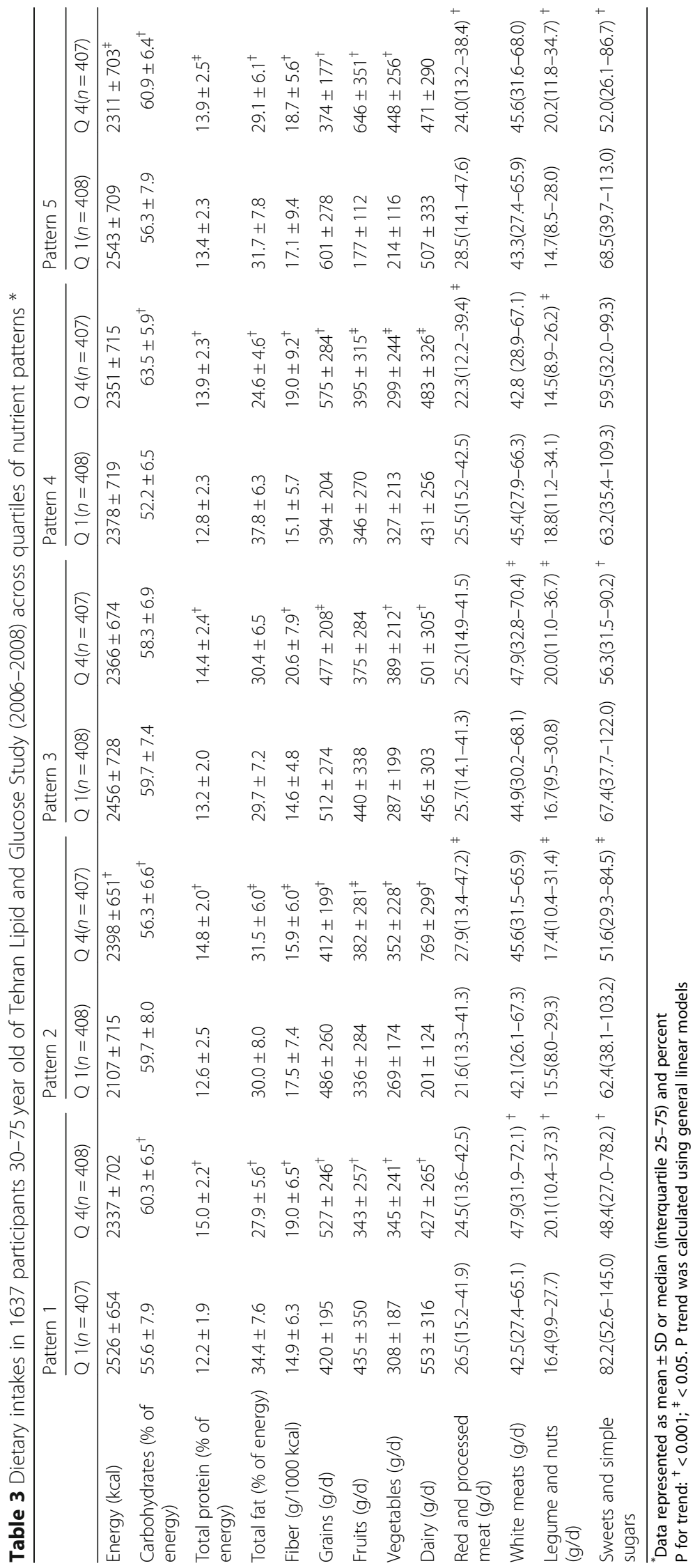


Table 4 Multiple linear regression analysis evaluating the association between each quartile increase of nutrient patterns and changes in fasting blood sugar, systolic and diastolic blood pressure after three years of follow up in 1637 participants of the Tehran Lipid and Glucose Study

\begin{tabular}{|c|c|c|c|c|c|c|}
\hline & \multicolumn{2}{|c|}{ Fasting blood sugar changes } & \multicolumn{2}{|c|}{ Systolic blood pressure changes } & \multicolumn{2}{|c|}{ Diastolic blood pressure changes } \\
\hline & $\beta^{a}(95 \% C l)$ & $P$ value & $\beta(95 \% C l)$ & $P$ value & $\beta(95 \% \mathrm{Cl})$ & $P$ value \\
\hline \multicolumn{7}{|l|}{ Pattern 1} \\
\hline Model $1^{\mathrm{b}}$ & $0.894(-0.056,1.843)$ & 0.065 & $-0.210(-0.750,0.323)$ & 0.444 & $-0.149(-0.532,0.234)$ & 0.446 \\
\hline Model2 ${ }^{c}$ & $0.791(-0.159,1.742)$ & 0.103 & $-0.336(-0.900,0.167)$ & 0.178 & $-0.264(-0.643,0.115)$ & 0.172 \\
\hline Model $^{\mathrm{d}}$ & $0.659(-0.298,1.615)$ & 0.177 & $-0.320(-0.817,0.176)$ & 0.206 & $-0.205(-0.550,0.140)$ & 0.243 \\
\hline \multicolumn{7}{|l|}{ Pattern 2} \\
\hline Model $1^{\mathrm{b}}$ & $-0.221(-1.163,0.720)$ & 0.645 & $-0.205(-0.743,0.334)$ & 0.456 & $-0.110(-0.492,0.273)$ & 0.574 \\
\hline Model2 ${ }^{c}$ & $-0.217(-1.157,0.723)$ & 0.651 & $-0.195(-0.726,0.337)$ & 0.472 & $-0.114(-0.492,0.263)$ & 0.552 \\
\hline Model ${ }^{d}$ & $-0.019(-0.973,0.937)$ & 0.968 & $-0.210(-0.710,0.291)$ & 0.411 & $-0.188(-0.536,0.160)$ & 0.289 \\
\hline \multicolumn{7}{|l|}{ Pattern 3} \\
\hline Model $1^{\mathrm{b}}$ & $0.146(-0.804,1.095)$ & 0.763 & $0.113(-0.429,0.655)$ & 0.683 & $0.056(-0.328,0.441)$ & 0.774 \\
\hline Model2 ${ }^{c}$ & $0.152(-0.795,1.099)$ & 0.753 & $0.100(-0.434,0.634)$ & 0.713 & $0.059(-0.328,0.431)$ & 0.789 \\
\hline Model $^{\mathrm{d}}$ & $0.140(-0.806,1.086)$ & 0.772 & $0.122(-0.372,0.615)$ & 0.629 & $0.068(-0.275,0.412)$ & 0.695 \\
\hline \multicolumn{7}{|l|}{ Pattern 4} \\
\hline Model $1^{b}$ & $0.064(-0.889,1.017)$ & 0.895 & $-0.174(-0.719,0.372)$ & 0.533 & $-0.150(-0.538,0.238)$ & 0.448 \\
\hline Model2 ${ }^{c}$ & $0.123(-0.828,1.074)$ & 0.800 & $-0.113(-0.651,0.425)$ & 0.680 & $-0.097(-0.479,0.286)$ & 0.620 \\
\hline Model ${ }^{\mathrm{d}}$ & $0.047(-0.904,0.999)$ & 0.922 & $-0.147(-0.644,0.351)$ & 0.563 & $-0.068(-0.414,0.279)$ & 0.702 \\
\hline \multicolumn{7}{|l|}{ Pattern 5} \\
\hline Model $1^{\mathrm{b}}$ & $-0.026(-0.988,0.935)$ & 0.957 & $0.085(-0.465,0.635)$ & 0.762 & $0.036(-0.354,0.426)$ & 0.857 \\
\hline Model ${ }^{C}$ & $-0.108(-1.069,0.853)$ & 0.825 & $-0.002(-0.545,0.540)$ & 0.993 & $-0.032(-0.417,0.354)$ & 0.871 \\
\hline Model ${ }^{d}$ & $-0.166(-1.128,0.796)$ & 0.735 & $-0.089(-0.592,0.413)$ & 0.727 & $-0.099(-0.448,0.250)$ & 0.579 \\
\hline
\end{tabular}

${ }^{a}$ Beta regression coefficient; the positive $\beta$ values indicated that higher adherence to nutrient patterns increases the changes in dependent variables and vice versa

${ }^{\mathrm{b}}$ Adjusted for age and sex

${ }^{c}$ Adjusted for model 1 and body mass index, physical activity, and smoking (yes or no)

${ }^{\mathrm{d}}$ Adjusted for model 2 and energy intake, education levels (under diploma, diploma and associate degree, bachelor and higher), marital status (single, married), and employment status (employed, unemployed). For blood pressure and fasting blood sugar changes, their corresponding values in baseline phase were adjusted

valuable to mention that a nutrient factor predicts a lower amount of cardiometabolic factor changes compared with main factors such as age, sex, and body mass index; also nutrient patterns have many interactions which attenuates or reinforces the overall effect of the pattern. If more nutrients in a pattern have similar direction in applying their physiological effects, the overall effect of the pattern is strengthening, and vice versa. Furthermore, mechanisms which regulate serum glucose and BP are highly sensitive, and maintain their levels in a normal range [22], in comparison to weight which is easily influenced by dietary energy and fat intakes. Although the association of single nutrient such as sodium, calcium, potassium, magnesium [23], and amino acids [21, 24] with BP, and simple sugars, fructose, etc. [25] with FBS has been proved previously; but when the association of whole nutrients with FBS and BP were evaluated, the interaction of useful, inactive and potentially harmful nutrients is inevitable and their effects may be neutralized by each other. Also in the general population most people have normal FBS and BP, while they consume all nutrients from their diet.

Nutrient pattern and prospective changes of lipid profiles In the current study, the fifth pattern, which was characterized by nutrients rich in fruit and vegetables, was inversely related to 3-year changes in TGs, while other patterns showed no significant relationship with serum levels of lipid profiles. At baseline, individuals became older and more obese across quartiles of the fifth pattern; however, they had lower levels of TGs. The positive role of the fifth pattern components including vitamin $\mathrm{A}$, vitamin $\mathrm{C}$, and high intake of fruit and vegetable in reducing the risk of metabolic syndrome was shown in Korean adults [26]. In Australian middle aged men, a nutrient pattern rich of vitamin $\mathrm{A}$ and antioxidant agents existing in fruit, had a significant reverse relation with CRP and inflammation [27]. The association of 
Table 5 Multiple linear regression analysis evaluating the association between each quartile increase of nutrient patterns and changes in lipid profile after three years of follow up in 1637 participants of the Tehran Lipid and Glucose Study

\begin{tabular}{|c|c|c|c|c|c|c|c|c|}
\hline & \multicolumn{2}{|l|}{ Triglyceride changes } & \multicolumn{2}{|c|}{ HDL-cholesterol changes } & \multicolumn{2}{|c|}{ LDL-cholesterol changes } & \multicolumn{2}{|l|}{ Total cholesterol } \\
\hline & $\beta^{\text {a }}(95 \%$ Cl) & $P$ value & $\beta^{\mathrm{a}}(95 \% \mathrm{Cl})$ & $P$ value & $\beta^{\text {a }}(95 \%$ Cl) & $P$ value & $\beta^{\mathrm{a}}(95 \% \mathrm{Cl})$ & $P$ value \\
\hline \multicolumn{9}{|l|}{ Pattern 1} \\
\hline Model $1^{\mathrm{b}}$ & $-1.16(-4.02,1.69)$ & 0.425 & $-0.11(-0.43,-0.19)$ & 0.471 & $-0.45(-1.59,0.68)$ & 0.436 & $-0.98(-2.25,0.29)$ & 0.131 \\
\hline Model $2^{c}$ & $-1.24(-4.11,1.62)$ & 0.395 & $-0.11(-0.42,-0.20)$ & 0.491 & $-0.30(-1.45,0.83)$ & 0.601 & $-0.85(-2.13,0.42)$ & 0.192 \\
\hline Model $3^{d}$ & $-1.28(-4.17,1.65)$ & 0.382 & $-0.11(-0.43,-0.20)$ & 0.476 & $-0.28(-1.43,0.86)$ & 0.627 & $-0.86(-2.15,0.42)$ & 0.191 \\
\hline \multicolumn{9}{|l|}{ Pattern 2} \\
\hline Model $1^{\mathrm{b}}$ & $1.37(-1.47,4.22)$ & 0.343 & $0.02(-0.28,0.33)$ & 0.871 & $0.71(-0.42,1.84)$ & 0.218 & $0.84(-0.42,2.11)$ & 0.191 \\
\hline Model $2^{c}$ & $1.46(-1.38,4.30)$ & 0.314 & $0.02(-0.29,0.33)$ & 0.898 & $0.76(-0.37,1.89)$ & 0.187 & $0.88(-0.38,2.15)$ & 0.172 \\
\hline Model3 ${ }^{d}$ & $1.77(-1.12,4.66)$ & 0.231 & $0.07(-0.24,0.39)$ & 0.661 & $0.62(-0.52,1.78)$ & 0.286 & $0.85(-0.44,2.15)$ & 0.195 \\
\hline \multicolumn{9}{|l|}{ Pattern 3} \\
\hline Model $1^{\mathrm{b}}$ & $-0.29(-3.16,2.57)$ & 0.839 & $-0.12(-0.43,0.19)$ & 0.454 & $0.27(-0.86,1.41)$ & 0.637 & $-0.03(-1.31,1.24)$ & 0.957 \\
\hline Model2 $2^{c}$ & $-0.32(-0.319,2.54)$ & 0.825 & $-0.11(-0.43,0.19)$ & 0.471 & $0.26(-0.87,1.40)$ & 0.647 & $-0.05(-1.33,1.22)$ & 0.937 \\
\hline Model3 ${ }^{d}$ & $-0.36(-3.23,2.50)$ & 0.802 & $-0.12(-0.44,0.18)$ & 0.430 & $0.29(-0.84,1.44)$ & 0.607 & $-0.03(-1.31,1.24)$ & 0.957 \\
\hline \multicolumn{9}{|l|}{ Pattern 4} \\
\hline Model1 ${ }^{b}$ & $0.03(-2.85,2.91)$ & 0.983 & $-0.08(-0.40,0.23)$ & 0.604 & $0.60(-0.54,1.75)$ & 0.303 & $0.86(-0.42,2.14)$ & 0.189 \\
\hline Model $2^{c}$ & $0.12(-2.75,3.00)$ & 0.931 & $-0.08(-0.40,0.23)$ & 0.605 & $0.58(-0.56,1.73)$ & 0.316 & $0.87(-0.40,2.16)$ & 0.181 \\
\hline Model $3^{d}$ & $0.02(-2.86,2.91)$ & 0.987 & $-0.10(-0.42,0.21)$ & 0.525 & $0.63(-0.51,1.79)$ & 0.278 & $0.89(-0.39,2.18)$ & 0.175 \\
\hline \multicolumn{9}{|l|}{ Pattern 5} \\
\hline Model $1^{\mathrm{b}}$ & $-3.40(-6.30,-0.49)$ & 0.022 & $0.16(-0.15,0.48)$ & 0.313 & $-0.71(-1.86,0.44)$ & 0.227 & $-0.96(-2.25,0.34)$ & 0.148 \\
\hline Model2 ${ }^{c}$ & $-3.51(-6.24,-0.60)$ & 0.018 & $0.16(-0.15,0.48)$ & 0.303 & $-0.61(-1.78,0.54)$ & 0.299 & $-0.88(-2.18,0.42)$ & 0.185 \\
\hline Model ${ }^{d}$ & $-3.66(-6.57,-0.74)$ & 0.014 & $0.14(-0.18,0.46)$ & 0.392 & $-0.56(-1.73,0.60)$ & 0.341 & $-0.88(-2.18,0.42)$ & 0.186 \\
\hline
\end{tabular}

${ }^{a}$ Beta regression coefficient; the positive $\beta$ values indicated that higher adherence to nutrient patterns increases the changes in dependent variables and vice versa

${ }^{\mathrm{b}}$ Adjusted for age and sex

cAdjusted for model 1 and body mass index, physical activity, and smoking (yes or no)

${ }^{\mathrm{d}}$ Adjusted for model 2 and energy intake, education levels (under diploma, diploma and associate degree, bachelor and higher), marital status (single, married), and employment status (employed, unemployed). For each of lipid profile factors, its corresponding value in baseline phase was adjusted

nutrient patterns and lipid profiles in the form of metabolic syndrome has been previously investigated [15]. Khayyatzadeh et al. indicated that patterns with high simple carbohydrates such as fructose, increased the level of TGs in women; however, patterns with high selenium, vitamin A and B groups decreased TG levels in men [15]. Fructose had a high factor loading in the fifth pattern of our study. The direct association of dietary fructose, consumed from high-fructose corn syrup and sweetened beverages, with TG levels observed [28, 29]. Review articles on clinical trials had shown that fructose in an isocaloric diet does not increase TG levels compared to other carbohydrates; however, if the calorie intake increases, fructose increases the synthesis of TG. Generally, there is no definitive finding that fructose necessarily increases TG levels compared to other carbohydrates [30, 31]. In our fifth pattern, fructose intake was mostly supplied by fruits, not beverages. The conservative effect of fruit and vegetable consumption on TGs levels has been proven. The other nutrient patterns had no significant relationship with changes in serum lipid profiles which may be due to interactions between nutrient components For example, in the third pattern, fiber can reduce the absorption of divalent metals including calcium, iron, and manganese [32]; these nutrients seem to attenuate the effects of each other. Whereas, nutrients of the fifth pattern, such as vitamin c, fructose and pantothenic acid, have boosting effects on some biological actions in the human body [33, 34].

What matters is founding nutrient patterns which significantly have beneficial association with cardiometabolic factors in epidemiological studies and then testing these patterns in different communities and consequently focusing on a nutrient pattern as complementary treatment for prevention of cardiometabolic complications.

The present study has valuable strengths, including the prospective design, relatively high sample size, and high accuracy in nutritional data measurement which was collected by trained and experienced dieticians compared with previous studies that mostly used selfreported questionnaires. There are also limitations to this study; despite the adjustment of many possible 
confounders, we do not rule out the possibility of unknown confounders affecting the finding of this study; also, since identification of patterns in this study was done using factor analysis, the limitations of this type of analysis can also be our research constraints. The number of factors derived from the factor analysis are largely influenced by the decisions of the researchers through the number of nutrients entering the analysis, which effect the factor loading of nutrients in each pattern, the method for obtaining the data matrix (rotation method or other methods) and selecting the number of factors (patterns) [9]. However, we selected a wide range of main nutrients which may have effects on cardiometabolic factors; also, five predominant patterns had explained $62.2 \%$ of the total variations in dietary intakes of main nutrients.

\section{Conclusions}

In summary, the findings of the present study showed that consuming a pattern of nutrients rich in vitamin $\mathrm{A}$, vitamin $\mathrm{C}$, pyridoxine, potassium and fructose or a pattern rich of nutrients originates from fruit and vegetable is associated with a reduction in serum TG.

\section{Abbreviations \\ TLGS: Tehran Lipid and Glucose Study; CVD: Cardiovascular diseases; BMI: Body mass index; BP: Blood pressure; FFQ: Food frequency questionnaire; USDA: United States Department of Agriculture; FCT: Food composition Table; WC: Waist circumference; TG: Triglyceride; HDL-C: High- density lipoprotein-cholesterol; LDL-C: Low-density lipoprotein-cholesterol; TC: Total cholesterol; FBS: Fasting blood sugar; PCA: Principal component analysis; 95\% Cl: 95\% Confidence interval}

\section{Acknowledgements}

The authors would like to thank the "Student Research Committee", "Research \& Technology Chancellor", and "Research Institute for Endocrine Sciences" in Shahid Beheshti University of Medical Sciences for their financial support of this study. The authors express their appreciation to staff of the Tehran Lipid and Glucose Study Unit of the Research Institute for Endocrine Sciences, for their valuable help. We would like to acknowledge Dr. Forough Ghanbari for critical editing of English grammar and syntax of the manuscript.

\section{Authors' contributions}

M.M. and P.S conceptualized and designed the study. F.T. and F.H.E. analysed and interpreted the data. M.M., P.S., F.T., F.H.E., and P.M. drafted the initial manuscript. P.M and F.A supervised the project and all authors approved the final version of the manuscript as submitted.

\section{Funding}

This study was supported by a grant of the student research committee; project NO [1396/46475], Shahid Beheshti University of Medical Sciences, Tehran, Iran. It was also supported by the Research Institute for Endocrine Sciences, Shahid Beheshti University of Medical Sciences, Tehran, Iran. These funders provided financial resources and skilled manpower for collecting the data.

\section{Availability of data and materials}

The datasets used and/or analysed during the current study are available from the corresponding author on reasonable request.

\section{Ethics approval and consent to participate}

The proposal of this study was approved by the Ethics Committee of the Research Institute for Endocrine Science, Shahid Beheshti University of
Medical Sciences, Tehran, Iran. An informed written consent was obtained from all participants.

\section{Consent for publication}

In the informed written consent, participants allowed to the researchers for publishing the results of the study.

\section{Competing interests}

The authors report no conflict of interest.

\section{Author details}

${ }^{1}$ Student Research committee, Department of Clinical Nutrition and Dietetics, Faculty of Nutrition and Food Technology, Shahid Beheshti University of Medical Sciences, Tehran, Iran. ${ }^{2}$ Department of Clinical Nutrition and Dietetics, Faculty of Nutrition and Food Technology, Shahid Beheshti University of Medical Sciences, Tehran, Iran. ${ }^{3}$ Nutrition and Endocrine Research Center, Research Institute for Endocrine Sciences, Shahid Beheshti University of Medical Sciences, P.O. Box: 1985717413, Tehran, Iran.

${ }^{4}$ Department of Nutrition, School of Public Health, Iran University of Medical Sciences, Tehran, Iran. ${ }^{5}$ Endocrine Research Center, Research Institute for Endocrine Sciences, Shahid Beheshti University of Medical Sciences, Tehran, Iran.

Received: 8 January 2020 Accepted: 23 April 2020

Published online: 11 May 2020

\section{References}

1. Clark H. NCDs: a challenge to sustainable human development. Lancet (London, England). 2013;381:510-1.

2. Roth GA, Johnson C, Abajobir A, et al. Global, regional, and National Burden of cardiovascular diseases for 10 causes, 1990 to 2015. J Am Coll Cardiol. 2017;70:1-25.

3. Grundy SM. Obesity, metabolic syndrome, and cardiovascular disease. J Clin Endocrinol Metab. 2004;89:2595-600.

4. NCD Risk Factor Collaboration (NCD-RisC). Worldwide trends in diabetes since 1980: a pooled analysis of 751 population-based studies with 4.4 million participants. Lancet (London, England). 2016;387:1513-30.

5. NCD Risk Factor Collaboration (NCD-RisC). Worldwide trends in body-mass index, underweight, overweight, and obesity from 1975 to 2016: a pooled analysis of 2416 population-based measurement studies in 128.9 million children, adolescents, and adults. Lancet (London, England). 2017;390:262742.

6. NCD Risk Factor Collaboration (NCD-RisC). Worldwide trends in blood pressure from 1975 to 2015: a pooled analysis of 1479 population-based measurement studies with 19.1 million participants. Lancet (London, England). 2017:389:37-55.

7. Farzadfar F, Finucane MM, Danaei G, et al. National, regional, and global trends in serum total cholesterol since 1980: systematic analysis of health examination surveys and epidemiological studies with 321 country-years and 3.0 million participants. Lancet (London, England). 2011;377:578-86.

8. Schwerin HS, Stanton JL, Smith JL, et al. Food, eating habits, and health: a further examination of the relationship between food eating patterns and nutritional health. Am J Clin Nutr. 1982;35:1319-25.

9. Hu FB. Dietary pattern analysis: a new direction in nutritional epidemiology. Curr Opin Lipidol. 2002;13:3-9.

10. Newby PK, Tucker KL. Empirically derived eating patterns using factor or cluster analysis: a review. Nutr Rev. 2004;62:177-203.

11. Gnagnarella P, Maisonneuve P, Bellomi M, et al. Nutrient intake and nutrient patterns and risk of lung cancer among heavy smokers: results from the COSMOS screening study with annual low-dose CT. Eur J Epidemiol. 2013; 28:503-11.

12. Pisa PT, Pedro TM, Kahn K, et al. Nutrient patterns and their association with socio-demographic, lifestyle factors and obesity risk in rural south African adolescents. Nutrients. 2015;7:3464-82.

13. Moskal A, Pisa PT, Ferrari P, et al. Nutrient patterns and their food sources in an international study setting: report from the EPIC study. PLoS One. 2014;9: e98647.

14. Salehi-Abargouei A, Esmaillzadeh A, Azadbakht L, et al. Nutrient patterns and their relation to general and abdominal obesity in Iranian adults: findings from the SEPAHAN study. Eur J Nutr. 2016;55:505-18. 
15. Khayyatzadeh SS, Moohebati M, Mazidi M, et al. Nutrient patterns and their; relationship to metabolic syndrome in Iranian adults. Eur J Clin Investig. 2016;46(10):840-52.

16. Chikowore T, Pisa PT, van Zyl T, et al. Nutrient Patterns Associated with Fasting Glucose and Glycated Haemoglobin Levels in a Black South African Population. Nutrients; 2017;9.19;9(1).

17. Freisling H, Pisa PT, Ferrari P, et al. Main nutrient patterns are associated with prospective weight change in adults from 10 European countries. Eur J Nutr. 2016;55:2093-104.

18. Azizi F, Ghanbarian A, Momenan AA, et al. Prevention of noncommunicable disease in a population in nutrition transition: Tehran lipid and glucose study phase II. Trials. 2009;10:5.

19. Mirmiran P, Hosseini-Esfahani F, Mehrabi Y, et al. Reliability and relative validity of an FFQ for nutrients in the Tehran Lipid and Glucose Study. Public Health Nutr. 2010;13(5):654-62

20. Teymoori F, Asghari G, Salehi P, et al. Are dietary amino acids prospectively predicts changes in serum lipid profile? Diabetes \& metabolic syndrome. 2019;13:1837-43.

21. Teymoori F, Asghari G. Dietary amino acids and incidence of hypertension: A principle component analysis approach, vol. 7; 2017. p. 16838.

22. Navar LG. The kidney in blood pressure regulation and development of hypertension. Med Clin North Am. 1997;81:1165-98.

23. Key minerals to help control blood pressure. It's usually best to get calcium, magnesium, and potassium from food. Are you getting enough? Harv Health Lett. 2014;39:5.

24. Teymoori F, Asghari G, Mirmiran P, et al. High dietary intake of aromatic amino acids increases risk of hypertension. J Am Soc Hypertension. 2017; 12(1):25-33.

25. Shambaugh $\mathrm{P}$, Worthington $\mathrm{V}$, Herbert JH. Differential effects of honey, sucrose and fructose on blood sugar levels. J Manip Physiol Ther. 1991;14: 91-2.

26. Park S, Ham J-O Lee B-K. Effects of total vitamin a, vitamin C, and fruit intake on risk for metabolic syndrome in Korean women and men. Nutrition. 2015; 31:111-8.

27. Cao Y, Wittert G, Taylor AW, et al. Nutrient patterns and chronic inflammation in a cohort of community dwelling middle-aged men. Clin Nutr (Edinburgh, Scotland). 2016;36(4):1040-7.

28. Mock K, Lateef S, Benedito VA, et al. High-fructose corn syrup-55 consumption alters hepatic lipid metabolism and promotes triglyceride accumulation. J Nutr Biochem. 2017;39:32-9.

29. Huang D, Dhawan T, Young S, et al. Fructose impairs glucose-induced hepatic triglyceride synthesis. Lipids Health Dis. 2011;10:20.

30. Chiavaroli L, de Souza RJ, Ha V, et al. Effect of fructose on established lipid targets: a systematic review and meta-analysis of controlled feeding trials. J Am Heart Assoc. 2015;4:e001700.

31. David Wang D, Sievenpiper JL, de Souza RJ, et al. Effect of fructose on postprandial triglycerides: a systematic review and meta-analysis of controlled feeding trials. Atherosclerosis. 2014;232:125-33.

32. Slavin JL. Position of the American dietetic association: health implications of dietary fiber. J Am Diet Assoc. 2008:108:1716-31.

33. Padayatt $S$, Daruwala R, Wang $Y$, et al. Vitamin C: from molecular actions to optimum intake. In: Handbook of Antioxidants CADENZAS, E; PACKER, I. 2nd ed. Washington DC: CRC press; 2001. p. 117-45.

34. Park SY, Murphy SP, Martin CL, et al. Nutrient intake from multivitamin/ mineral supplements is similar among users from five ethnic groups: the multiethnic cohort study. J Am Diet Assoc. 2008;108:529-33.

\section{Publisher's Note}

Springer Nature remains neutral with regard to jurisdictional claims in published maps and institutional affiliations.

Ready to submit your research? Choose BMC and benefit from:

- fast, convenient online submission

- thorough peer review by experienced researchers in your field

- rapid publication on acceptance

- support for research data, including large and complex data types

- gold Open Access which fosters wider collaboration and increased citations

- maximum visibility for your research: over $100 \mathrm{M}$ website views per year

At $\mathrm{BMC}$, research is always in progress.

Learn more biomedcentral.com/submissions 